\title{
BMJ Open Variations in outcomes by residential location for women with breast cancer: a systematic review
}

\author{
Paramita Dasgupta, ${ }^{1}$ Peter D Baade, ${ }^{1,2,3}$ Danny R Youlden, ${ }^{1}$ Gail Garvey, ${ }^{4}$ \\ Joanne F Aitken, ${ }^{1,5,6}$ Isabella Wallington, ${ }^{7}$ Jennifer Chynoweth, ${ }^{7}$ Helen Zorbas, ${ }^{7}$ \\ Philippa H Youl ${ }^{1,2,5}$
}

To cite: Dasgupta P, Baade PD, Youlden DR, et al. Variations in outcomes by residential location for women with breast cancer: a systematic review. BMJ Open 2018;8:e019050. doi:10.1136/ bmjopen-2017-019050

- Prepublication history and additional material for this paper are available online. To view these files, please visit the journal online (http://dx.doi. org/10.1136/bmjopen-2017019050).

Received 8 August 2017 Revised 14 December 2017 Accepted 14 February 2018

Check for updates

${ }^{1}$ Cancer Research Centre, Cancer Council Queensland,

Brisbane, Queensland, Australia

${ }^{2}$ None, Menzies Health Institute Queensland, Griffith University, Brisbane, Queensland, Australia

${ }^{3}$ School of Mathematical

Sciences, Queensland University of Technology, Brisbane, Queensland, Australia

${ }^{4}$ Menzies School of Health

Research, Brisbane, Queensland, Australia

${ }^{5}$ School of Public Health and

Social Work, Queensland

University of Technology,

Brisbane, Queensland, Australia

${ }^{6}$ Institute for Resilient Regions,

University of Southern

Queensland, Toowoomba,

Queensland, Australia

${ }^{7}$ Cancer Australia, Sydney, New

South Wales, Australia

Correspondence to

Dr Peter D Baade;

peterbaade@cancerqld.org.au

\section{ABSTRACT}

Objectives To systematically assess the evidence for variations in outcomes at each step along the breast cancer continuum of care for Australian women by residential location.

Design Systematic review.

Methods Systematic searches of peer-reviewed articles in English published from 1 January 1990 to 24 November 2017 using PubMed, EMBASE, CINAHL and Informit databases. Inclusion criteria were: population was adult female patients with breast cancer; Australian setting; outcome measure was survival, patient or tumour characteristics, screening rates or frequencies, clinical management, patterns of initial care or post-treatment follow-up with analysis by residential location or studies involving non-metropolitan women only. Included studies were critically appraised using a modified NewcastleOttawa Scale.

Results Seventy-four quantitative studies met the inclusion criteria. Around $59 \%$ were considered high quality, 34\% moderate and $7 \%$ low. No eligible studies examining treatment choices or post-treatment follow-up were identified. Non-metropolitan women consistently had poorer survival, with most of this differential being attributed to more advanced disease at diagnosis, treatment-related factors and socioeconomic disadvantage. Compared with metropolitan women, non-metropolitan women were more likely to live in disadvantaged areas and had differing clinical management and patterns of care. However, findings regarding geographical variations in tumour characteristics or diagnostic outcomes were inconsistent.

Conclusions A general pattern of poorer survival and variations in clinical management for Australian female patients with breast cancer from non-metropolitan areas was evident. However, the wide variability in data sources, measures, study quality, time periods and geographical classification made direct comparisons across studies challenging. The review highlighted the need to promote standardisation of geographical classifications and increased comparability of data systems. It also identified key gaps in the existing literature including a lack of studies on advanced breast cancer, geographical variations in treatment choices from the perspective of patients and post-treatment follow-up.

\section{INTRODUCTION}

Worldwide, breast cancer is the most frequently diagnosed cancer among women,
Strengths and limitations of this study

- First systematic review examining evidence for geographical variations in breast cancer outcomes across the continuum of care for Australian women.

- Review was conducted according to published guidelines.

- All included articles were subject to quality assessment.

- Wide heterogeneity across studies in study quality, levels of evidence, methodology, data sources, time period and terminology.

No meta-analysis was possible.

accounting for $25 \%$ of all new diagnoses in 2012, and is the leading cause of female cancer mortality $(15 \%$ of total cancer deaths). ${ }^{1}$ Among Australian women, breast cancer is also the most common cancer and the second leading cause of cancer mortality. ${ }^{2}$ Like other developed countries, Australia has high breast cancer incidence rates but relatively low-mortality rates ${ }^{1}$ with significant and ongoing improvements in survival, most likely due to earlier detection, screening mammography and improved treatments. ${ }^{3}$ However, not all women have benefited equally from these improvements with international studies consistently reporting geographical variations in survival ${ }^{4-6}$ and across the breast cancer continuum of care (such as screening, diagnosis, treatment, post-treatment and psychosocial care).$^{7-9}$ While Australia has relatively high-survival rates compared with international benchmarks ${ }^{10}$ significant variations exist with poorer survival for rural and disadvantaged women. ${ }^{11} 12$

Australia has a universal healthcare system; however, it is also a country of vast distances with cancer-related services typically being concentrated in major cities ${ }^{13}$ so that those living elsewhere often face long travel times and limited access to specialised care. ${ }^{11} 14$ 
Although about $20 \%$ of the total Australian population live outside a major city, for some states and territories, this percentage increases to over one-third. ${ }^{15}$ There is also considerable overlap between remoteness and socioeconomic status; around one-third of the population living in major cities in Australia also live in areas classified as least disadvantaged, compared with only $2 \%$ of those from very remote areas. ${ }^{16}$ Current strategies to better address the needs of rural patients with cancer and to make cancer care more accessible include the Australian Government's establishment of cancer centres and radiation facilities in regional Australia, exploring innovative models of care and other local-level initiatives. ${ }^{14} 17$

A comprehensive understanding of the drivers of variations in outcomes across population groups is a prerequisite for ensuring equitable cancer care and improving outcomes for all Australians. This systematic review aimed to identify, assess and synthesise the current evidence relating to geographical variations in survival, patient and tumour characteristics and diagnostic and clinical outcomes for female Australian patients with breast cancer. It was conducted as part of a larger systematic review that also investigated psychosocial outcomes ${ }^{18}$ and variations by indigenous status. ${ }^{19}$ Such a review may help identify gaps in knowledge, formulate strategic research priorities and develop evidence-based interventions to reduce the observed inequities.

\section{METHODS}

\section{Terminology}

Due to the range of definitions used to define geographical areas, geographical remoteness was categorised into 'metropolitan' areas (typically 'major cities' or 'urban') and 'non-metropolitan' areas (comprising the remaining localities). However, where relevant, important patterns observed within the remoteness categories were described in greater detail such as studies relating specifically to remote or very remote areas.

\section{CLINICAL QUESTIONS}

The published Preferred Reporting Items for Systematic Reviews and Meta-Analyses (PRISMA) guidelines for conducting systematic reviews ${ }^{20}$ were followed for this review. As a first step, a series of clinical questions to guide the review were clearly defined and agreed on before commencing the review process in consultation with a Project Steering Group that included clinicians, researchers, allied health practitioners, consumer advocates with experience in breast cancer and health policy representatives. All questions conformed to PICO guidelines ${ }^{20}$ in which the target population $(\mathrm{P})$, intervention/ exposure (I), comparator $(\mathrm{C})$ and outcomes $(\mathrm{O})$ are clearly defined and used to guide the review process, with the comparator being the only optional component. ${ }^{21}$

Eleven clinical questions examining variations between non-metropolitan and metropolitan women with breast
Box 1 Clinical questions guiding the systematic review

\section{Survival outcomes}

- In women diagnosed with breast cancer, do non-metropolitan women have poorer breast cancer survival compared with metropolitan women in Australia?

\section{Patient and tumour characteristics}

- In women diagnosed with breast cancer, do non-metropolitan women have different sociodemographic characteristics compared with metropolitan women in Australia?

- In women diagnosed with breast cancer, do non-metropolitan women have more advanced tumour characteristics compared with metropolitan women in Australia?

\section{Diagnostic and treatment outcomes}

- In women diagnosed with breast cancer, are non-metropolitan women in the breast screening target group less likely to access breast screening services compared with metropolitan women in Australia?

- In women diagnosed with breast cancer, are non-metropolitan women in the breast screening target group less likely to adhere to recommended breast screening intervals ( 2 yearly) compared with metropolitan women in Australia?

- In women diagnosed with breast cancer, are there differences in the clinical management between non-metropolitan and metropolitan women in Australia?

- In women diagnosed with breast cancer, are non-metropolitan women less likely to receive the recommended clinical management compared with metropolitan women in Australia?

- In women diagnosed with breast cancer, are non-metropolitan women more likely to experience delays in referral to breast cancer specialist clinicians compared with metropolitan women in Australia?

- In women diagnosed with breast cancer, do non-metropolitan women experience fewer treatment options compared with metropolitan women in Australia?

- In women diagnosed with breast cancer, are non-metropolitan women less likely to complete prescribed treatment compared with metropolitan women in Australia?

- In women diagnosed with breast cancer, are non-metropolitan women less likely to participate in recommended follow-up compared with metropolitan women in Australia?

cancer (collectively referred to as 'residential location') were grouped according to (1) survival (one question), (2) patient/tumour characteristics (two questions) and (3) diagnostic and treatment outcomes (eight questions) (box 1).

\section{LITERATURE SEARCHES}

The electronic databases: PubMed, EMBASE, CINAHL and Informit were systematically searched for all indexed articles from 1 January 1990 to 24 November 2017. The Web of Science database was used for cited reference searches.

Search strategies were based on keywords and subject headings to reflect the review aim with separate queries designed for each clinical question (see online supplementary appendix 1). Key terms of 'breast neoplasms', 'female' and 'Australia' were combined with terms 
relating to geographical aspects including 'rural health', 'geographic inequalities', 'spatial', 'health services accessibility' and 'remoteness' and outcome measures of interest notably 'survival', 'stage', 'diagnosis age', 'socioeconomic', 'mammography', 'screening rate', 're-screening', 'clinical management', 'patterns of care', 'mastectomy', 'breast reconstruction', 'chemotherapy', 'radiotherapy', 'lymph node' and 'guideline adherence'. Additional synonyms reflecting each of the key terms were also included.

\section{INCLUSION CRITERIA}

Studies were eligible if they met the following inclusion criteria:

1. the population included adult female patients with breast cancer or focused on a breast cancer-specific subgroup

2. had an Australian setting

3. the outcome measure was survival, patient or tumour characteristics, screening participation or frequency, clinical management, patterns of initial care or post-treatment follow-up

4. was

a. a quantitative study on non-metropolitan versus metropolitan comparisons

b. a qualitative study on geographical inequalities

c. quantitative or qualitative studies reporting on relevant outcomes for non-metropolitan women only.

The scope of the review was limited to English language peer-reviewed original research articles. Reviews, editorials, books, conference abstracts and commentaries were excluded, although when identified through the systematic searches their reference lists were examined for relevant articles.

\section{REVIEW PROCESS}

After removing duplicates, the titles and abstracts of all articles identified during the searches were independently reviewed by two authors (first PD, second PHY, DRY or PDB) for possible inclusion based on their relevance to each clinical question. Discrepancies were clarified through discussion between the two reviewers, and if necessary, the other reviewers were consulted. Full-text versions of all articles of potential relevance were then retrieved for more detailed independent assessment by two reviewers as before. During this process, articles were classified as 'include' or 'exclude' with reasons for exclusion being documented. Reviewer decisions were compared, and any disagreements resolved by consensus.

\section{CRITICAL APPRAISAL}

The quality of all included articles was critically assessed by two independent reviewers using the NewcastleOttawa Scale (NOS), ${ }^{22}$ a risk of bias assessment tool for non-randomised studies recommended by the Cochrane Collaboration $^{23}$ that can be readily tailored for the critical appraisal of quantitative cohort studies. ${ }^{9}$ The NOS assesses studies on six items over five broad perspectives: (1) selection bias, (2) measurement of confounders, (3) outcome assessment, (4) follow-up and (5) adjustments for residual confounders (two items). We extended this tool by incorporating features from other published checklists $^{2425}$ to include three additional items to assess (1) study attrition (missing data), (2) statistical methods and (3) data presentation. Studies were scored according to the extent that they met each of the nine assessed criterion (see online supplementary appendix 2) using an ordinal scale to rate the risk of bias as 0 (high), 1 (intermediate) and 2 (low), and the individual item scores then summed to give a total quality score. Instances of major differences in total scores between the two reviewers for individual articles were resolved by consensus, and each article was then assigned a summary score (averaged across the two scores). The total average score (range of $0-18)$ achieved across the nine criterion was categorised as 'high' (14-18), 'moderate' (9-13.5) or 'low' $(<9)$ quality. Studies were not excluded based specifically on their quality rating.

Studies were also classified according to the published levels of evidence for quantitative observational studies from the Australian National Health and Medical Research Council ${ }^{24}$ in decreasing order of strength as Level I, Level II, Level III-1, Level III-2, Level III-3 or Level IV.

\section{DATA EXTRACTION}

For all included articles, study characteristics including author(s), publication year, title, population, design and outcomes were recorded in a customised database by one reviewer and subsequently checked by another. Any errors or inconsistencies were resolved after consulting the original source.

\section{RESULTS}

\section{Study selection}

The steps in the review process are illustrated in a PRISMA diagram (figure 1). A total of 476 articles were identified across combined databases with an additional 45 citations from other sources. After removing duplicates, an initial pool of 211 articles remained of which 65 were excluded after initial scanning of title/abstracts. Of the 146 retrieved full-text articles, 74 met the inclusion criteria and were considered relevant to at least one of the clinical questions. Excluded studies are listed in online supplementary appendix 3, including reasons for exclusion.

\section{STUDY CHARACTERISTICS}

All included articles were quantitative, and around $80 \%$ used administrative data sources such as population-based 


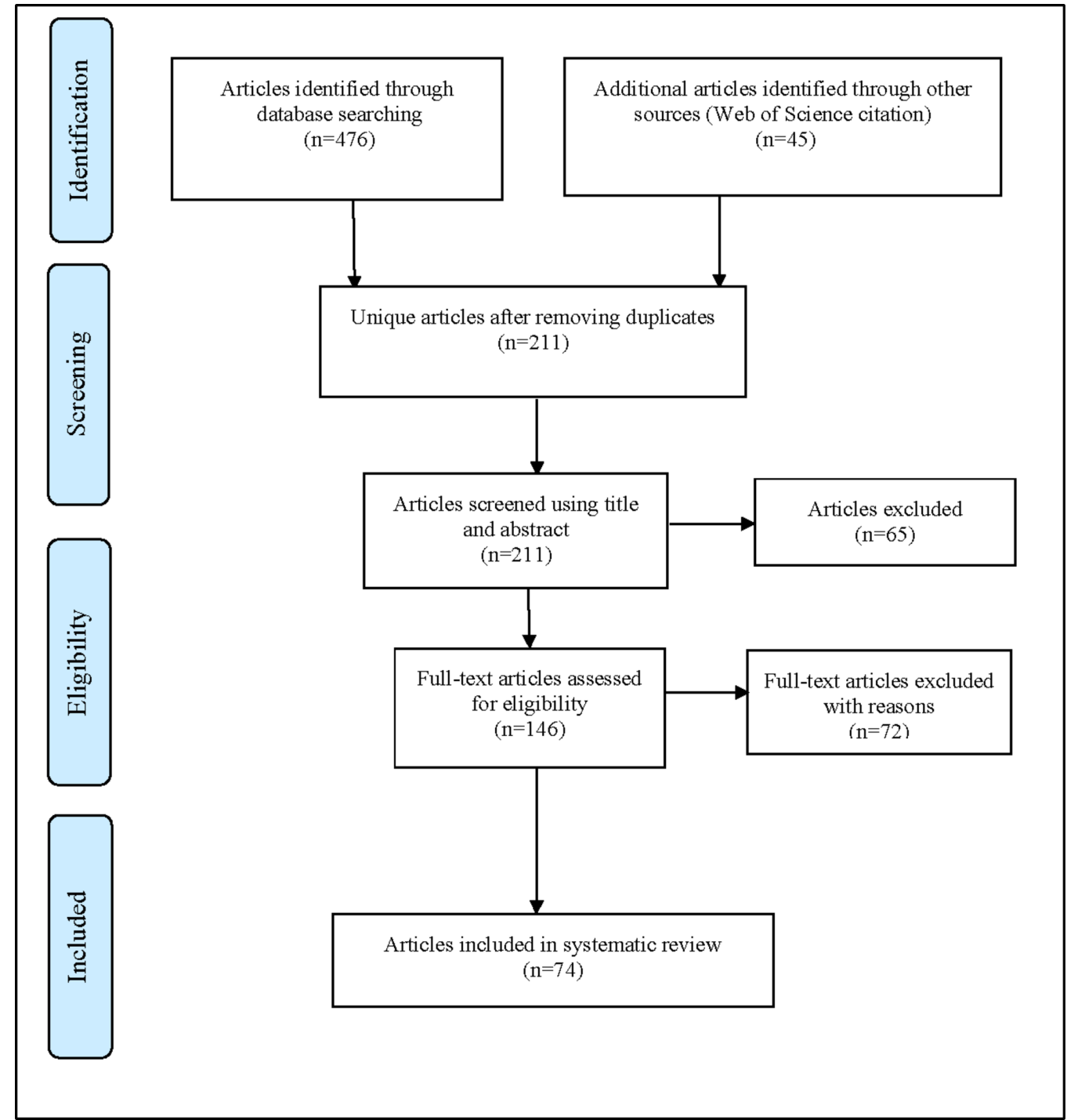

Figure 1 Process of inclusion and exclusion of studies for the systematic review.

cancer registries, screening databases or the non-representative (not population based) National Breast Cancer Audit database which has collected data on about $60 \%$ of invasive early breast cancers treated by participating Australian (and New Zealand) breast surgeons since $1998{ }^{26}$ Remaining studies were based on medical record reviews and cross-sectional surveys.

There was considerable heterogeneity in the definition of non-metropolitan and metropolitan populations. While more than half $(57 \%)$ of the included studies used standardised definitions such as the Rural, Remote and Metropolitan Areas system, the Accessibility/Remoteness Index of Australia (ARIA) or ARIA+ or remoteness areas defined by the Australian Standard Geographical Classification, ${ }^{27}$ others defined non-metropolitan and metropolitan areas based on distances to services, population density or postcodes. Two studies did not provide detailed information regarding the basis of their geographical classification (table 1).

Around $59 \%$ of included studies were graded as high quality, $34 \%$ moderate and $7 \%$ low quality, with a mean score of 13.0 and range of 6.5-17.0. Key limiting factors for these scores were that around one-third $(30 \%)$ of studies did not use a population-based representative sample, whereas $20 \%$ did not adjust for confounders (including age and sociodemographics). Studies based on reliable and objective data sources (cancer registries) were limited in their ability to control for clinical and treatment factors. The use of highly selective or convenience samples and lack of follow-up also reduced study quality. No studies provided Level I evidence, whereas more than half $(57 \%)$ gave Level II evidence, $34 \%$ Level III-3 and 9\% Level IV evidence (table 1).

\section{KEY FINDINGS}

Studies are summarised below (tables 2-5, also online supplementary appendix 4) according to clinical questions within each of the key themes: (1) survival outcomes, (2) patient/ tumour characteristics and (3) diagnostic and treatment outcomes. Several studies reported on multiple outcomes. The emphasis is on whether there was evidence of variations 
Table 1 Summary scores, overall grades and levels of evidence for included studies

\begin{tabular}{|c|c|c|c|c|}
\hline Study & Metropolitan/non-metropolitan definition & Score* $^{*}$ & Quality† & Levelł \\
\hline Adelson et al, $1997^{73}$ & Based on health services & 15 & High & III-3 \\
\hline Ahern et al, $2015^{84}$ & $\mathrm{ARIA+}$ & 7 & Low & IV \\
\hline $\begin{array}{l}\text { Australian Institute of Health and } \\
\text { Welfare, } 2013^{12}\end{array}$ & $\mathrm{ARIA+}$ & 14.5 & High & II \\
\hline Baade et al, $2016^{77}$ & Distance to radiation treatment facilities & 16 & High & II \\
\hline Barratt et al, $1997^{62}$ & RRMA Classification & 9.5 & Moderate & II \\
\hline Bell et al, $2012^{88}$ & Postcodes§ & 15 & High & II \\
\hline Bonett et al, $1990^{29}$ & Postcodes§ & 14.5 & High & II \\
\hline Clayforth et al, $2007^{31}$ & Postcodes§ & 15 & High & II \\
\hline Cockburn et al, $1997^{67}$ & NA: rural and remote women only & 10 & Moderate & III-3 \\
\hline Chong et al, $2015^{85}$ & ASGC & 13 & Moderate & III-3 \\
\hline Collins et al, $2017^{101}$ & NA: regional women only & 14 & High & II \\
\hline Craft et al, $1997^{78}$ & RRMA Classification & 12 & Moderate & III-3 \\
\hline Cramb et al, $2012^{32}$ & Distance to radiation treatment facilities & 15.5 & High & II \\
\hline Cramb et al, $2016 a^{34}$ & ASGC & 14 & High & II \\
\hline Cramb et al, $2016 b^{33}$ & ASGC & 15 & High & II \\
\hline Eley et al, $2008^{96}$ & NA: rural and remote women only & 7.5 & Low & IV \\
\hline Flitcroft et al, $2016^{90}$ & $\mathrm{ARIA+}$ & 10 & Moderate & III-3 \\
\hline Fox et al, $2013^{55}$ & RRMA Classification & 10.5 & Moderate & III-3 \\
\hline Hall and Holman, $2003^{92}$ & ARIA & 14.5 & High & II \\
\hline Hall et al, $2004 a^{37}$ & ARIA & 15 & High & II \\
\hline Hall et al, 2004b $b^{80}$ & ARIA & 14.5 & High & II \\
\hline Hill et al, $1994^{79}$ & Postcodes§ & 12.5 & Moderate & II \\
\hline Hsieh et al, $2013^{48}$ & $\mathrm{ARIA+}$ & 14 & High & II \\
\hline Hsieh et al, $2015^{83}$ & Distance to radiation treatment facilities & 14 & High & II \\
\hline Hsieh et al, 2016a ${ }^{38}$ & ASGC & 14 & High & II \\
\hline Hsieh et al, 2016b ${ }^{47}$ & $\mathrm{ARIA+}$ & 15 & High & II \\
\hline Hughes et al, $2014^{68}$ & Postcodes§ & 10.5 & Moderate & III-3 \\
\hline Kok et al, $2006^{53}$ & RRMA Classification & 14.5 & High & III-3 \\
\hline Koshy et al, $2005^{81}$ & Postcodes§ & 9.5 & Moderate & III-3 \\
\hline Kricker et al, $2001^{82}$ & Unclear & 16 & High & II \\
\hline Lai et al, $2007^{93}$ & RRMA Classification & 15 & High & II \\
\hline Lam et al, $2015^{100}$ & NA: regional women only & 10 & Moderate & IV \\
\hline
\end{tabular}


Table 1 Continued

\begin{tabular}{|c|c|c|c|c|}
\hline Study & Metropolitan/non-metropolitan definition & Score $^{*}$ & Quality† & Levelf \\
\hline Leung et al, $2014^{60}$ & ARIA+ & 12.5 & Moderate & III-3 \\
\hline Leung et al, $2015^{61}$ & ARIA+ & 12 & Moderate & III-3 \\
\hline Leung et al, $2016^{57}$ & ARIA+ & 13 & Moderate & III-3 \\
\hline Lord et al, $2012^{56}$ & ARIA & 14 & High & $\|$ \\
\hline Luke et al, $2004^{58}$ & Postcodes§ & 14 & High & $\|$ \\
\hline Martin et al, $2006^{74}$ & Based on residential area & 14.5 & High & ॥ \\
\hline Mastaglia and Kristjanson, $2001^{75}$ & Unclear & 6.5 & Low & IV \\
\hline Mitchell et al, $2006^{39}$ & Postcodes§ & 16 & High & $\|$ \\
\hline Morris et al, $2012^{87}$ & ASGC & 10.5 & Moderate & III-3 \\
\hline O'Byrne et al, $2000^{69}$ & RRMA Classification & 15.5 & High & III-3 \\
\hline Ristevski et al, $2012^{98}$ & NA: regional women only & 9 & Moderate & IV \\
\hline Roder et al, $2012 \mathrm{a}^{28}$ & ASGC & 14.5 & High & III-3 \\
\hline Roder et al, 2012b $b^{108}$ & ASGC & 14 & High & III-3 \\
\hline Roder et al, 2013a ${ }^{49}$ & ASGC & 14 & High & III-3 \\
\hline Roder et al, 2013b 26 & ASGC & 14.5 & High & III-3 \\
\hline Roder et al, 2013c ${ }^{91}$ & ASGC & 14.5 & High & III-3 \\
\hline Roder et al, $2014^{50}$ & ASGC & 15 & High & III-3 \\
\hline Schofield et al, $1994^{65}$ & Distance to screening services & 10.5 & Moderate & II \\
\hline Siahpush and Singh, $2002^{66}$ & Based on residential area & 12.5 & Moderate & II \\
\hline Spilsbury et al, $2005^{40}$ & Postcodes§ & 16 & High & ॥ \\
\hline Sullivan et al, $2003^{63}$ & Postcodes§ & 11 & Moderate & III-3 \\
\hline Supramaniam et al, $2014^{41}$ & ARIA+ & 17 & High & II \\
\hline Taylor, $1997^{46}$ & Capital city, other metropolitan, rural & 14.5 & High & II \\
\hline Tervonen et al, $2017^{42}$ & ARIA+ & 14 & High & II \\
\hline Thompson et al, $2008^{76}$ & ARIA+ & 14.5 & High & II \\
\hline Tracey et al, $2008^{43}$ & ARIA & 15 & High & $\|$ \\
\hline Tulloh and Goldsworthy, $1997^{99}$ & NA: rural and remote women only & 7 & Low & III-3 \\
\hline Weber et al, $2014^{64}$ & ARIA+ & 10.5 & Moderate & III-3 \\
\hline Wilkinson and Cameron, $2004^{45}$ & Postcodes§ & 9.5 & Moderate & $\|$ \\
\hline Yu et al, $2015^{44}$ & ARIA+ & 12 & Moderate & II \\
\hline
\end{tabular}

${ }^{*}$ Average score over scores from two independent reviewers. Please refer to text for further details.

†Quality categories: high (score 14-18), moderate (score 9-13.5) or low (score <9). Please refer to text for further details.

$\ddagger$ Australian National Health and Medical Research Council ${ }^{24}$ levels of evidence in decreasing order of strength are Level I, Level II, Level III-1, Level III-2, Level III-3 and Level IV.

§Postcodes within state capital were considered metropolitan, remaining were non-metropolitan.

ARIA, Accessibility/Remoteness Index of Australia; ASGC, Australian Standard Geographical Classification; NA, not applicable; RRMA, Rural,

Remote and Metropolitan Area.

in relevant outcomes by residential location and, if so, the direction and a quantitative estimate of the magnitude of the effect. Given the considerable heterogeneity among studies in terms of their quality, levels of evidence, time period and geographical definitions, we have deliberately interpreted any summary patterns with caution.

\section{SURVIVAL OUTCOMES}

There was a consistent pattern of significantly poorer survival (in unadjusted analyses) for women in non-metropolitan areas compared with metropolitan women across 21 (19 high and 2 moderate quality) of 22 included studies both nationally ${ }^{12} 28$ and at the state level (table 3). ${ }^{29-45}$ The 5-year unadjusted relative survival for female breast cancers was about 2\%-5\% (absolute) lower for non-metropolitan than metropolitan women. The one exception was an early highquality study involving women in New South Wales (diagnosed from 1980 to 1991) who did not report any survival differential. ${ }^{46}$

However, no geographical differential in survival was evident across $11^{28} 32363739-434647$ of 20 studies that also 
Table 2 Summary of included studies on differentials in breast cancer survival outcomes by residential location

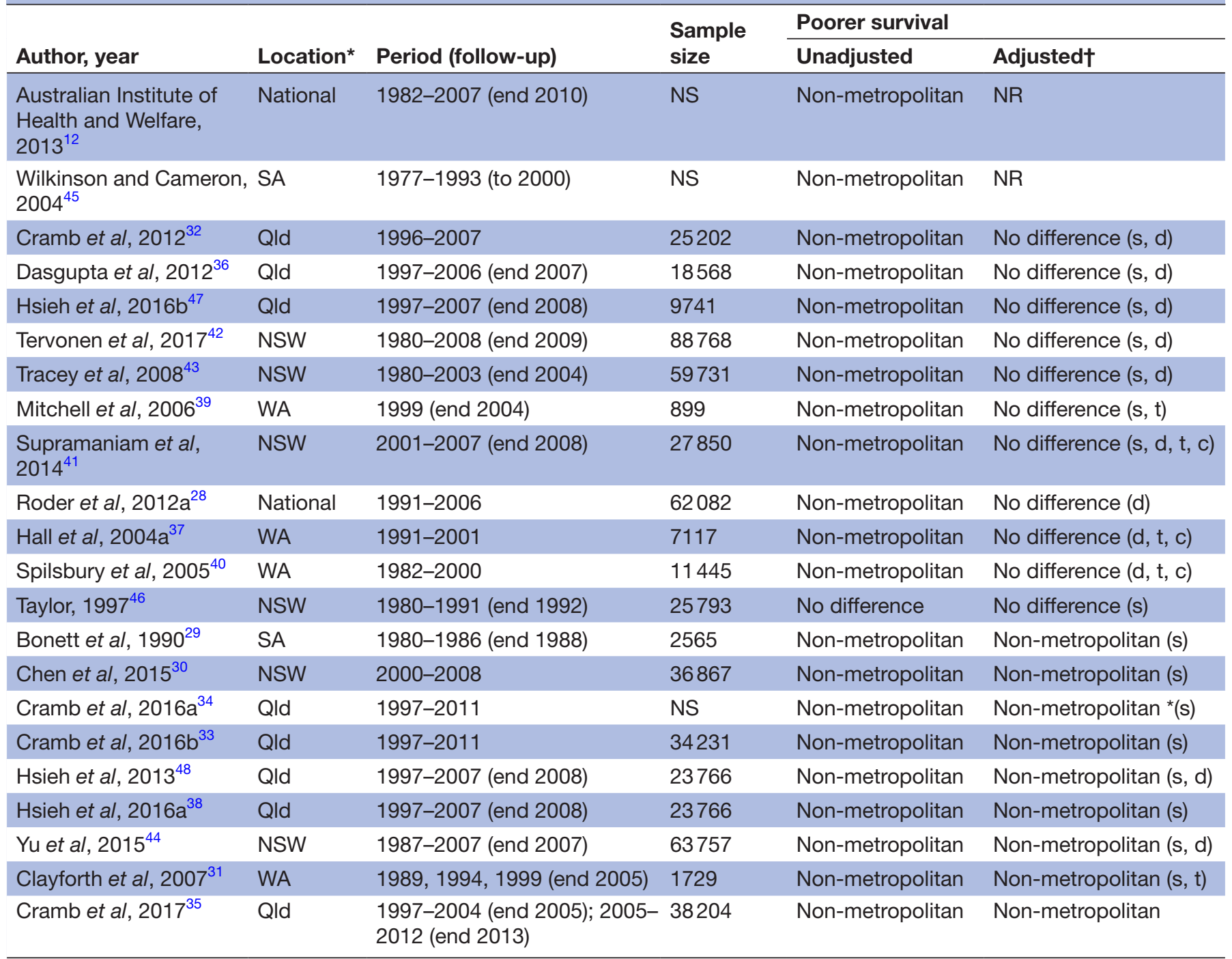

*National: all states/territories. NSW, New South Wales; Qld, Queensland; SA, South Australia; WA, Western Australia.

$\dagger$ †ll adjusted for age except Bonett et al, 1990. ${ }^{29}$

(s) Also adjusted for some measure of spread of diagnosis, such as stage at diagnosis or tumour diameter.

(t) Also adjusted for treatment-related factors.

(d) Also adjusted for area disadvantage.

(c) Also adjusted for comorbidities.

NR, not relevant; NS, not stated.

reported survival estimates after adjustment for various combinations of known survival determinants including demographics, area-level disadvantage, spread of disease, comorbidities and treatment-related factors. The remaining nine studies ${ }^{29-31} 33-354448$ all reported poorer survival for non-metropolitan women even after adjustment.

The adjusted results varied according to the combination of variables included in the statistical models. Six of the seven papers that reported significant differentials after adjusting for a measure of stage at diagnosis did not consider comorbidities or treatment-related factors. ${ }^{29} 3033343844$ Of the five studies that adjusted for treatment-related factors, four reported no evidence of a survival differential, ${ }^{37}{ }^{39-41}$ whereas the finding of a significant difference was likely to be limited to women diagnosed prior to the mid-1990s in the remaining study. ${ }^{31}$

Most of the 22 included studies focused on medium-term survival, with only one ${ }^{43}$ following women for longer than 5 years after their breast cancer diagnosis.

\section{PATIENT AND TUMOUR CHARACTERISTICS Patient characteristics}

Both of the included high-quality studies that reported a positive association between area-disadvantage and non-metropolitan residence were based on analysis of 30299 early invasive female breast cancer cases from the National Breast Cancer Audit (table 4) ${ }^{49}{ }^{50}$ For example, 
Table 3 Summary of included studies on differentials in patient and tumour characteristics by residential location

\begin{tabular}{|c|c|c|c|c|}
\hline Author, year & Location* & Period & Sample size & Findingt \\
\hline \multicolumn{5}{|c|}{ Patient characteristics (higher area-level socioeconomic disadvantage) } \\
\hline Roder et al, $2013 a^{49}$ & National & 1998-2010 & 30299 & Non-metropolitan \\
\hline \multicolumn{5}{|c|}{ Tumour characteristics (higher spead of disease) ${ }^{3}$} \\
\hline Bonett et al, $1990^{29}$ & SA & 1980-1986 & 1171 & No difference \\
\hline Fox et al, $2013^{55}$ & NSW & 2008-2011 & 400 & No difference \\
\hline Leung et al, $2016^{57}$ & NSW, Qld, Vic & 1997-2011 & 195 & No difference \\
\hline Lord et al, $2012^{56}$ & NSW & 2001-2002 & 6664 & No difference \\
\hline Luke et al, $2004^{58}$ & SA & 1997-2002 & 4912 & No difference \\
\hline Mitchell et al, $2006^{39}$ & WA & 1999 & 899 & No difference \\
\hline Kok et al, $2006^{53}$ & Vic & 1993-2000 & 5294 & Non-metropolitan \\
\hline Roder et al, 2013b26 & National & 1998-2010 & 30299 & Non-metropolitan \\
\hline Tracey et al, $2008^{43}$ & NSW & 1980-2003 & 59731 & Metropolitan \\
\hline
\end{tabular}

*National: all states/territories. NSW, New South Wales; QId, Queensland; SA, South Australia; Vic, Victoria; WA, Western Australia.

†Some measure of spread of disease such as stage at diagnosis or tumour size.

Table 4 Summary of included studies on differentials in diagnostic outcomes by residential location

\begin{tabular}{|c|c|c|c|c|}
\hline Author, year & Location* & Period & Sample size† & Finding \\
\hline \multicolumn{5}{|l|}{ Higher screening ratef } \\
\hline Barratt et al, $1997^{62}$ & National & 1996 & 1035 & No difference \\
\hline Leung et al, $2015^{61}$ & National & 2010 & 10011 & No difference \\
\hline Sullivan et al, $2003^{63}$ & WA & $1982-2000$ & 380 & Non-metropolitan \\
\hline Schofield et al, $1994^{65}$ & Vic & 1988-1990 & 668 & Metropolitan \\
\hline $\begin{array}{l}\text { Siahpush and Singh, } \\
2002^{66}\end{array}$ & National & 1995 & 10179 & Metropolitan \\
\hline Cockburn et al, $1997^{67}$ & Vic & 1995 & $\begin{array}{l}180 \text { non- } \\
\text { metropolitan }\end{array}$ & $\begin{array}{l}\text { No screening history and knowing } \\
\text { service locations screening predictors }\end{array}$ \\
\hline Leung et al, $2015^{61}$ & National & 2010 & 10011 & Non-metropolitan \\
\hline O’Byrne et al, $2000^{69}$ & Vic & 1995-1996 & 121889 & Non-metropolitan \\
\hline $\begin{array}{l}\text { Siahpush and Singh, } \\
2002^{66}\end{array}$ & National & 1995 & 10179 & Metropolitan \\
\hline
\end{tabular}

${ }^{*}$ National: all states/territories. NSW, New South Wales; Vic, Victoria; WA, Western Australia.

†Only aged 50-69 years who were eligible at time of this review and all included studies for publicly funded BreastScreen programme in Australia.

†Having had at least one screening mammogram.

$\S$ Having another screening mammogram within 2 years of the initial screen. 
Table 5 Summary of included studies on differentials in treatment outcomes by residential location

\begin{tabular}{|c|c|c|c|c|}
\hline Author, year & Location* & Period & Sample size & Finding \\
\hline \multicolumn{5}{|l|}{ Higher mastectomy } \\
\hline Koshy et al, $2005^{81}$ & NSW, ACT & $1997-2002$ & 1069 & No difference \\
\hline Kricker et al, $2001^{82}$ & NSW & 1992,1995 & 2020 or 2883 & No difference \\
\hline Adelson et al, $1997^{73}$ & NSW & 1991-1992 & 4038 & Non-metropolitan \\
\hline Azzopardi et al, $2014^{72}$ & National & 1998-2012 & 21643 & Non-metropolitan \\
\hline Martin et al, $2006^{74}$ & WA & 1990-1999 & 2713 & Non-metropolitan \\
\hline $\begin{array}{l}\text { Mastaglia and Kristjanson, } \\
2001^{75}\end{array}$ & WA & 1996-1997 & 160 & Non-metropolitan \\
\hline Roder et al, 2013b 26 & National & $1998-2010$ & 30299 & Non-metropolitan \\
\hline Thompson et al, $2008^{76}$ & Qld & 2004-2005 & 1274 & Non-metropolitan \\
\hline \multicolumn{5}{|c|}{ Higher breast-conserving surgery } \\
\hline Hall et al, $2004 b^{80}$ & WA & $1991-2000$ & 7304 & No difference \\
\hline Adelson et al, $1997^{73}$ & NSW & 1991-1992 & 4038 & Metropolitan \\
\hline Azzopardi et al, $2014^{72}$ & National & 1998-2012 & 21643 & Metropolitan \\
\hline Baade et al, $2016^{77}$ & Qld & $1997-2011$ & 11631 & Metropolitan \\
\hline Craft et al, $1997^{78}$ & National & 1993 & 4683 & Metropolitan \\
\hline Hill et al, $1994^{79}$ & Vic & 1990 & 856 & Metropolitan \\
\hline Kok et al, $2006^{53}$ & Vic & $1993-2000$ & 5294 & Metropolitan \\
\hline Mitchell et al, $2006^{39}$ & WA & 1999 & 899 & Metropolitan \\
\hline Roder et al, $2013 a^{49}$ & National & $1998-2010$ & 30299 & Metropolitan \\
\hline \multicolumn{5}{|l|}{ Lower radiotherapy } \\
\hline Azzopardi et al, $2014^{72}$ & National & 1998-2012 & 21643 & Non-metropolitan \\
\hline Hsieh et al, $2015^{83}$ & Qld & $1997-2008$ & 6357 & Non-metropolitan \\
\hline Kok et al, $2006^{53}$ & Vic & $1993-2000$ & 5294 & Non-metropolitan \\
\hline Mitchell et al, $2006^{39}$ & WA & 1999 & 899 & Non-metropolitan \\
\hline Hsieh et al, $2013 a^{48}$ & National & $1998-2010$ & 30299 & Non-metropolitan \\
\hline \multicolumn{5}{|l|}{ Lower hormonal therapy } \\
\hline Ahern et al, $2015^{84}$ & National & 2013 & 325 & No difference \\
\hline Hsieh et al, $2015^{83}$ & Qld & $1997-2008$ & 6357 & No difference \\
\hline Mitchell et al, $2006^{39}$ & WA & 1999 & 899 & Non-metropolitan \\
\hline \multicolumn{5}{|l|}{ Lower chemotherapy } \\
\hline Hsieh et al, $2015^{83}$ & Qld & $1997-2008$ & 6357 & No difference \\
\hline Hill et al, $1994^{79}$ & Vic & 1990 & 856 & No difference \\
\hline Mitchell et al, $2006^{39}$ & WA & 1999 & 899 & No difference \\
\hline Roder et al, $2013 a^{49}$ & National & $1998-2010$ & 30299 & Metropolitan \\
\hline \multicolumn{5}{|l|}{ Lower sentinel node biopsy } \\
\hline Chong et al, $2015^{85}$ & National & $2008-2010$ & 18737 & Non-metropolitan \\
\hline Dasgupta et al, 2017b ${ }^{86}$ & Qld & $\begin{array}{l}\text { July 2008- } \\
\text { December } 2012\end{array}$ & 5577 & Non-metropolitan \\
\hline Morris et al, $2012^{87}$ & National & 2008 (last 6 months) & 1267 to & Non-metropolitan \\
\hline \multicolumn{5}{|l|}{ Lower axillary surgery } \\
\hline Craft et al, $1997^{78}$ & National & 1993 & 4683 & No difference \\
\hline Kricker et al, $2001^{82}$ & NSW & 1992,1995 & 2020 or 2883 & No difference \\
\hline Thompson et al, $2008^{76}$ & Qld & 2004-2005 & 1274 & No difference \\
\hline
\end{tabular}

Continued 
Table 5 Continued

\begin{tabular}{|c|c|c|c|c|}
\hline Author, year & Location* & Period & Sample size & Finding \\
\hline Hall and Holman, $2003^{92}$ & WA & $1991-2000$ & 7303 & No difference \\
\hline Bell et al, $2012^{88}$ & Vic & 2004-2006 & 366 & Non-metropolitan \\
\hline Dasgupta et al, 2017c ${ }^{89}$ & Qld & $1997-2012$ & 4104 & Non-metropolitan \\
\hline Flitcroft et al, $2016^{90}$ & National & 2013 & 3786 & Non-metropolitan \\
\hline Roder et al, 2013c ${ }^{91}$ & National & 1998-2010 & 12207 & Non-metropolitan \\
\hline \multicolumn{5}{|l|}{ Higher unplanned admisisons } \\
\hline Lai et al, $2007^{93}$ & WA & 1995-1999 & 2703 & Non-metropolitan \\
\hline \multicolumn{5}{|l|}{ Access breast care nurses } \\
\hline Ahern et al, $2016^{94}$ & National & 2013 & 902 & No difference \\
\hline Campbell et al, $2006^{95}$ & National & 1997 & 544 & No difference \\
\hline \multicolumn{5}{|l|}{ Longer treatment delays } \\
\hline Fox et al, $2013^{55}$ & NSW & 2008-2011 & 400 & Non-metropolitan \\
\hline \multicolumn{5}{|l|}{ Poorer quality of care } \\
\hline Hill et al, $1994^{79}$ & Vic & 1990 & 856 & Non-metropolitan \\
\hline Fox et al, $2013^{55}$ & NSW & 2008-2011 & 400 & Non-metropolitan \\
\hline Baade et al, $2016^{77}$ & Qld & $1997-2011$ & 11631 & Non-metropolitan \\
\hline Mitchell et al, $2006^{39}$ & WA & 1999 & 899 & Non-metropolitan \\
\hline Roder et al, $2013 a^{49}$ & National & $1998-2010$ & 30299 & Non-metropolitan \\
\hline Roder et al, 2013b 26 & National & $1998-2010$ & 30299 & Non-metropolitan \\
\hline \multicolumn{5}{|l|}{ Treatment completion } \\
\hline Fox et al, $2013^{55}$ & NSW & 2008-2011 & 400 & $\begin{array}{l}\text { Non-metropolitan more likely to } \\
\text { complete chemotherapy }\end{array}$ \\
\hline Roder et al, 2012b $b^{108}$ & National & 1998-2005 & 36775 & $\begin{array}{l}\text { Non-metropolitan less likely to follow } \\
\text { clinician-recommended treatments }\end{array}$ \\
\hline \multicolumn{5}{|l|}{ Non-metropolitan } \\
\hline Budden et al, $2014^{97}$ & Qld & NS & 104 & $\begin{array}{l}\text { High-level satisfaction with treatment } \\
\text { decisions }\end{array}$ \\
\hline Eley et al, $2008^{96}$ & Qld & 2005-2006 & 51 & $\begin{array}{l}\text { Breast cancer nurses important } \\
\text { source of care }\end{array}$ \\
\hline Ristevski et al, $2012^{98}$ & Vic & NS & 70 & $\begin{array}{l}\text { High-level satisfaction with treatment } \\
\text { decisions }\end{array}$ \\
\hline Tulloh and Goldsworthy, $1997^{99}$ & Vic & 1992-1995 & 28 & $\begin{array}{l}\text { Rural setting did not influence quality } \\
\text { of care }\end{array}$ \\
\hline Lam et al, $2015^{100}$ & NSW & 2010-2014 & 574 & $\begin{array}{l}\text { A locally available publicly funded } \\
\text { radiotherapy service increased breast- } \\
\text { conserving surgery uptake }\end{array}$ \\
\hline Collins et al, $2017^{101}$ & Vic & 2009-2014 & 1213 & $\begin{array}{l}\text { Access to radiotherapy impacts } \\
\text { surgical management }\end{array}$ \\
\hline
\end{tabular}

*National: all states/territories. NSW, New South Wales; ACT, Australian Capital Territory; Qld, Queensland; Vic, Victoria; WA, Western Australia.

NS, not stated.

compared with affluent women, socioeconomically disadvantaged women diagnosed with breast cancer were 17 times more likely to live in remote areas (than metropolitan areas), ${ }^{49}$ whereas compared with metropolitan women, those from remote areas were 13 times more likely to live in a disadvantaged rather than more advantaged region. ${ }^{50}$

\section{Tumour characteristics}

No consistent pattern of variations in tumour characteristics by residential location was evident across the 13 included studies (table 4). Nationally, one high-quality study found that non-metropolitan women were $15 \%$ more likely to present with tumours $>40 \mathrm{~mm}$ (vs $<30 \mathrm{~mm}$ ), ${ }^{26}$ whereas three state-based high-quality studies also reported 
similar patterns, ${ }^{51-53}$ despite using different definitions of advanced disease. However, eight others (four high and four moderate quality) showed no differences, ${ }^{29} 3945$ 54-58 and one (high quality) that metropolitan women were $11 \%$ more likely to present with regional disease than non-metropolitan patients, but equally likely to present with distant tumours. $^{43}$

\section{Diagnostic and treatment outcomes}

Studies described here assessed geographical variations in relation to two broad topics: breast cancer screening (table 4) and treatment (table 5). The target group for the two screening questions refers to women aged 50 to 69 years who were eligible (at the time of this review) for the free population-based national mammographic programme in Australia (BreastScreen Australia). ${ }^{59}$

\section{Screening rate}

All eight of the included moderate quality studies relate to the publicly funded BreastScreen programme, as there were no data available to assess variations in private mammography, and provided mixed results. Analyses of self-reported data for more than 10000 women nationally found that despite poorer access to mammography services, ${ }^{60}$ non-metropolitan women had similar screening rates to metropolitan women, ${ }^{60}{ }^{61}$ consistent with an earlier cross-sectional survey ${ }^{62}$ Two state-based studies, however, reported higher participation rates in the BreastScreen programme for non-metropolitan women ${ }^{6364}$ In contrast, women who lived within $10-20 \mathrm{~km}$ of a relocatable BreastScreen service were $43 \%$ less likely to have been screened than those residing within $2 \mathrm{~km}$ of the service. ${ }^{65}$ Another study found that non-metropolitan women in the target age group were $39 \%$ more likely to report never having been screened through BreastScreen Australia than metropolitan women. ${ }^{66}$ Screening history, perceived breast cancer risk and knowledge about service location were among key predictors of accessing a relocatable screening service in a study involving only 180 non-metropolitan women. ${ }^{67}$

\section{Rescreening}

Results were inconsistent across the five included studies, with a dependence on the time period of data collection. One early (moderate quality) study showed that metropolitan women had higher rescreening rates through the national BreastScreen programme than non-metropolitan women, ${ }^{66}$ whereas among four other studies from 1995 onwards, one (moderate quality) study showed no difference in rescreening rates ${ }^{68}$ and three studies (two moderate and one high quality) showed that non-metropolitan women had higher rescreening rates. ${ }^{6069}$

\section{Clinical management}

Given there are separate Australian guidelines for clinical management of early ${ }^{70}$ and advanced stage breast cancer, ${ }^{71}$ the descriptions of variations in clinical management are categorised accordingly.
A consistent pattern of variations in the clinical management of early breast cancer by residential location was evident across 21 ( 14 high, 6 moderate and 1 low quality) of 28 included studies with 7 ( 3 high, 2 moderate and 2 low) finding no variations.

Among 30299 cases extracted from the National Breast Cancer Audit database, non-metropolitan women were at least five times more likely to have a mastectomy than metropolitan women, ${ }^{26}$ whereas another study using this database reported that the proportion of mastectomies progressively increased with increasing remoteness. ${ }^{72}$ Various state-specific studies also reported similar patterns. ${ }^{73-76}$ Studies using the National Breast Cancer Audit database found that non-metropolitan women were $6 \%$ less likely to undergo breast-conserving surgery, ${ }^{48}$ and that the proportion who had breast-conserving surgery decreased progressively with increasing remoteness. ${ }^{72}$ Similar findings were evident across six other state-level studies. ${ }^{39} 53$ 73 77-79 Only three studies reported no differences in surgical patterns by residential location. ${ }^{80-82}$

Two studies based on the National Breast Cancer Audit Database reported that non-metropolitan women were up to $20 \%$ less likely to receive adjuvant radiotherapy than metropolitan women. ${ }^{48} 72$ Moreover, women residing in areas lacking radiotherapy facilities had a higher likelihood $(23 \%)$ of not receiving radiotherapy than those from regions with such facilities. ${ }^{72}$ Three state-based studies also reported similar patterns. ${ }^{39} 5383$ Findings for other treatment modalities were less consistent with no geographical differentials in receipt of either hormonal therapy ${ }^{83}$ or chemotherapy, ${ }^{39} 7983$ higher uptake of chemotherapy $^{50}$ and lower for hormonal therapy among non-metropolitan women ${ }^{39}$ being reported.

Non-metropolitan women were consistently (12\%$58 \%$ ) less likely to undergo sentinel node biopsies $(\mathrm{SNBs})^{85-87}$ or postmastectomy breast reconstruction ${ }^{88-91}$ with only one earlier study reporting no difference in reconstruction rates. ${ }^{92}$ They also had a $10 \%$ higher risk of unplanned readmissions. ${ }^{93}$ However, no geographical variations in axillary node surgery ${ }^{7678} 82$ or access to specialist breast care nurses were evident. ${ }^{94} 95$

Of the seven included studies comprising non-metropolitan women only, one reported that breast care nurses were important in ensuring continuity of care,${ }^{96}$ two found a high level of patient satisfaction with the treatment decision process ${ }^{97} 98$ and one found that geographical setting was no impediment to receiving breast-conserving surgery or to accessing multidisciplinary care at a single non-metropolitan treatment centre. ${ }^{99}$ Among regional women in the state of New South Wales, breast-conserving surgical rates increased by $9 \%$ after a publicly funded radiotherapy service became available in 2013, compared with earlier years when the only options were a local private or publicly funded out-of-areas service. ${ }^{100}$ However, regional women who lived $\geq 100-200 \mathrm{~km}$ away (vs $<100 \mathrm{~km}$ ) from a radiotherapy service were twice as likely to have a mastectomy. ${ }^{101}$ 
The only study examining geographical variations in clinical management for advanced breast cancer found no geographical variations in mastectomy rates among women with metastatic disease. ${ }^{73}$

\section{Recommended clinical management}

Nine ( 4 high and 5 moderate quality) of 15 included studies reported geographical variations in guideline-concordant care with non-metropolitan women being less likely to undergo adjuvant radiotherapy, ${ }^{483} 7283$ hormonal therapy ${ }^{39}$ or $\mathrm{SNBs}^{85-87}$ and more likely to experience longer delays in commencing adjuvant chemotherapy. ${ }^{55}$ However, the other six studies (two high, two moderate and two low quality) found no significant geographical variations in receipt of recommended care. $^{767879828494}$

\section{Referral}

Non-metropolitan women were less likely to be referred to a radiation oncologist ${ }^{79}$ and were more likely to experience delays in assessment by a medical oncologist. ${ }^{55}$ Further, in a cross-sectional survey of 70 non-metropolitan women, $42 \%$ were referred to another health professional before surgery. ${ }^{98}$ All studies were of moderate quality.

International studies have consistently shown geographical variations in access to high-volume surgical care ${ }^{102-104}$ and provided clear evidence that such care is related to improved breast cancer survival ${ }^{103} 105$ and better concordance with clinical care guidelines. ${ }^{106} 107$ Hence, eligible studies that described access to high caseload surgeons were also considered for this clinical question. One highquality study reported that non-metropolitan women were $9 \%$ more likely to be treated locally by low caseload surgeons ${ }^{26}$ (defined as $\leq 10$ or $<20$ cases/year) with similar findings reported by three other high-quality studies. ${ }^{394977}$

\section{Treatment completion}

Of the two included studies, one found that non-metropolitan women were more likely to complete prescribed chemotherapy than metropolitan women. ${ }^{55}$ Another reported that women treated by low caseload surgeons ( $\leq 20$ cases/year) were more likely to decline clinician-recommended surgery, radiotherapy or chemotherapy based on data from the National Breast Cancer Audit. ${ }^{108}$

The review did not identify any studies examining geographical variations in the specific treatment options offered to non-metropolitan and metropolitan Australian female patients with breast cancer or post-treatment follow-up according to current national guidelines. ${ }^{109}$

\section{DISCUSSION}

This review found consistent evidence for variations in survival and clinical management, limited evidence for variations in diagnostic outcomes and inconsistent evidence for variations in tumour characteristics by residential location of Australian female patients with breast cancer.

While gaps in the literature limited our ability to draw clear links between identified variations and the drivers of these variations, there was good evidence that poorer breast cancer survival (at least up to 5 years after diagnosis) for non-metropolitan women reflects more advanced disease at diagnosis, greater comorbidities, treatment-related factors and area-level disadvantage. ${ }^{28} \quad 32 \quad 36 \quad 39-43$ According to the recent systematic review by the International Agency for Research on Cancer, ${ }^{110}$ there is sufficient evidence for the efficacy of mammographic screening in reducing breast cancer mortality for women aged 50 to 69 years. In Australia, increasing participation for groups with low screening rates can be achieved through the existing and well established population-based national mammographic programme (BreastScreen). Targeted strategies are required including thorough engagement and communication with primary care to improve screening participation rates. ${ }^{59}$ It is possible that these survival patterns are impacted by the lead time caused by mammographic screening, ${ }^{111}$ whereas we found only limited evidence that participation in the publicly funded BreastScreen services varies by geographical area, the lack of data on the number of privately screened women precludes an evaluation of actual population-based screening participation and its impact on the observed survival patterns. Hence, it remains a priority to explore means to combine data on public and private screening to gain more comprehensive information on total rates of breast cancer screening nationally.

The review found a consistent pattern of geographical variations in patterns of care and lower receipt of optimal clinical management for early breast cancer among non-metropolitan women in Australia. Reasons for these variations likely included limited access to oncological services and multidisciplinary care. ${ }^{112113}$ Regional Cancer Centres across Australia and integrated cancer networks were established to improve access to oncological care for regional patients. ${ }^{14}{ }^{17}$ However, overcoming barriers to multidisciplinary care, considered best practice in breast cancer care, ${ }^{114-116}$ in regional areas remains a challenge. Multidisciplinary cancer teams (MDTs) are sparse outside metropolitan areas and vary widely in the disciplines represented within existing teams. ${ }^{113}$

The efficacy of MDTs in informed clinical decision making, coordinated care and evidence-based practice for patients with breast cancer has been well documented. ${ }^{117-120}$ Several of the included studies in this review identified limited access to MDT care for non-metropolitan women as a possible contributor to lower receipt of guideline concordant care. ${ }^{26} 44498691108$ It is possible that the major benefits of MDT lie, in part, with greater adherence to standard therapy, ${ }^{44} 108119$ which may indirectly impact clinical outcomes.

The evidence for the impact of MDT on breast cancer survival is more limited, possibly reflecting methodological limitations and heterogeneity in MDT definitions. ${ }^{119} 121$ 
However, surgical specialisation has been shown to be associated with improved survival, ${ }^{105}$ and we found that non-metropolitan women had consistently poorer access to high-volume surgeons ${ }^{26} 394977$ which in Australia are predominantly based in major cities. ${ }^{122}$

Australian clinical practice guidelines for the management of early breast cancer recommend postoperative radiotherapy after breast-conserving surgery to reduce the risk of local recurrence, adjuvant endocrine therapy and/ or chemotherapy where appropriate based on hormone receptor status, ${ }^{123}$ and SNB offered to women with unifocal clinically node negative tumours $(\leq 30 \mathrm{~mm}) .{ }^{124}$ However, this review found limited but consistent evidence for geographical variations in receipt of care according to these guidelines. Specifically, non-metropolitan women were less likely to undergo adjuvant radiotherapy, ${ }^{3949} 537283$ hormonal therapy ${ }^{39}$ or SNB. ${ }^{85-87}$ Lower utilisation of SNB in non-metropolitan areas may reflect inadequate access to necessary resources, less relevant training and experience in performing SNB among general surgeons outside major treatment centres ${ }^{86} 87$ and lack of interdisciplinary collaboration required to perform SNBs. ${ }^{85-87}$ Surgeon-level interventions may be required to help improve SNB rates and hence quality of care and reduced morbidity.

The finding that non-metropolitan women were less likely to receive adjuvant radiotherapy likely reflects variations in access to such facilities. ${ }^{7283100101}$ However, it should be acknowledged that all included studies were published in the period 1 January 1990 to 24 November 2017, and that some earlier studies may not reflect current practice and/or the impact of improved access to radiation services with the development of new radiotherapy infrastructure in regional Australia over the last 5 years. ${ }^{1425}$ Both service affordability and availability impact radiotherapy utilisation ${ }^{126}$ with the uptake of breast-conserving surgery among regional women increasing after provision of a publicly funded local radiotherapy service. ${ }^{100}$ Similar patterns were also reported for radiotherapy utilisation among all regional patients with cancer. ${ }^{127} 128$ The waiting time from radiation oncologist assessment to receiving radiotherapy (for any cancer) has also improved over time. ${ }^{126}$ Although implementation of routine reporting of waiting times from diagnosis to commencing radiotherapy by geographical location would help identify when and where delays in referral and commencing treatment occur.

Given the potential survival benefits of adjuvant radiotherapy, ${ }^{129} 130$ the lower utilisation of radiotherapy among non-metropolitan women ${ }^{39} 485372$ and those with poorer access to radiotherapy facilities ${ }^{72} 83101$ is of concern.

Although some recent Queensland-based studies found limited evidence for a temporal reduction in geographical variations for breast cancer stage $e^{52}$ and surgical patterns, ${ }^{77}$ in practice, these changes were subtle and although the non-metropolitan to metropolitan differential reduced, it was still evident in the most recent time period. Moreover, despite improvements in survival over all areas in Queensland over time, geographical inequalities remained. ${ }^{33} 35$ These studies highlight the importance of ongoing monitoring of measured outcomes along breast cancer continuum to assess whether there has been a definitive change in these variations and to identify key drivers of any changes.

While the review found consistent evidence for variations in breast cancer survival and clinical management, patterns were inconsistent for other outcomes, primarily due to heterogeneity of the included studies or in some cases a lack of studies. These findings emphasise the importance of the work of Cancer Australia (Australia's national cancer control agency) in establishing a national comprehensive system for recording breast cancer stage and clinical management at the population level, thereby enabling accurate monitoring of the effectiveness of strategies and initiatives to improve breast cancer outcomes for non-metropolitan women in Australia.

On an international scale, inequities in access to specialised care $\mathrm{e}^{102-104}$ and geographical variations across the breast cancer continuum including screening, ${ }^{7}$ stage at diagnosis ${ }^{9} 131$ and patterns of care ${ }^{8107131-136}$ are well documented. There is widespread consensus that these variations reflect a combination of socioeconomic, demographic and environmental factors including geography, comorbidities, access, treatment and stage at diagnosis that defy easy solutions..$^{7-9} 103104131135$ The persistence of such inequities even for universal (publicly funded) healthcare systems ${ }^{7103131133136}$ highlights the complexity of the underlying issues.

\section{LIMITATIONS}

A number of issues made direct comparisons and to some extent interpretation of findings across studies particularly challenging. The assessment of comparability was hampered by the wide variability in study quality, levels of evidence, methodology, data sources, time period and terminology. These issues also prevented meta-analyses being carried out. Many studies were predominantly conducted at the state level, making the generalisation of findings to the national level difficult. The review also highlighted the need to improve and standardise definitions of geographical location to produce more uniform and reliable remoteness classifications. This would improve data comparability in terms of residential location and hence facilitate more definitive conclusions to be drawn on the strength of the available evidence. Similar concerns have been noted by international reviews on area-level variations in other cancer outcomes. ${ }^{8137138}$

Moreover, many studies had important limitations including selection bias and inadequate follow-up that impacted their quality. While using registry data allows generalisability of findings, such studies cannot comprehensively control for all potential confounders, especially those related to individual-level socioeconomic status, clinical or treatment factors since Australian cancer registries do not routinely collect information on these 
measures. ${ }^{139}$ Hence, population-based studies can adjust for area-level socioeconomic status but not between-persons differences. Only cross-sectional studies, although deemed inferior to population-based studies in terms of representativeness, can collect information on individual-level measures.

Considerable efforts were made to conduct a comprehensive search of existing literature on specified clinical questions by searching multiple databases with complex queries and evaluating reference lists of identified articles, published reviews and government reports to find additional articles. However, it is still possible that the search term criteria used could have unintentionally resulted in exclusion of relevant articles. Included articles were also limited to those indexed in the accessed databases.

\section{CONCLUSIONS}

By examining the current evidence relating to geographical variations in breast cancer outcomes across the continuum of care for Australian women, this review has important implications for clinical practice, service delivery and future research. It has highlighted the gap in knowledge of variations in the treatment of advanced breast cancers, patient decision making and post-treatment follow-up.

While addressing the geographical variations in breast cancer survival and clinical management will require a multifaceted approach, initial efforts could include improving access to and participation in breast screening programmes, raising awareness of the benefits of early detection and enabling all women diagnosed with breast cancer to be assessed by a multidisciplinary team that considers all relevant treatment options and have access to best practice treatment. To achieve equitable access for all women, it is crucial to promote coordinated care among non-metropolitan women and initiatives to facilitate the educational diffusion of healthcare changes among clinicians and patients through emerging technologies $^{140}$ to overcome barriers of distance. Recognising the heterogeneity of existing studies in terms of geographical coverage and definitions, the establishment of a national comprehensive system for recording breast cancer stage and clinical management would enable accurate monitoring of the success of these initiatives.

Finally, encouraging evidence-based research aimed at better understanding the reasons for geographical variations in breast cancer management and outcomes at each stage of the continuum of care needs to be a priority to inform the development of targeted initiatives to improve survival and quality of life for rural and remote women with breast cancer in Australia.

Acknowledgements The authors would like to acknowledge the advice of the Project Steering Committee.

Contributors All authors contributed to the design of the study. PHY and PDB coordinated the study. PD conducted the literature searches and drafted the manuscript. PD, PHY, DRY and PDB acted as reviewers and participated in data collection. PHY, PDB, DRY, JFA and GG contributed to the initial draft of the manuscript. All authors refined and approved the final version of the paper.

Funding This project was funded by Cancer Australia. PHY and GG are funded by a National Health and Medical Research Council Early Career Fellowship (nos 1054038 and 1105399 , respectively).

Competing interests None declared.

Patient consent Not required.

Provenance and peer review Not commissioned; externally peer reviewed.

Data sharing statement No additional data are available.

Open Access This is an Open Access article distributed in accordance with the Creative Commons Attribution Non Commercial (CC BY-NC 4.0) license, which permits others to distribute, remix, adapt, build upon this work non-commercially, and license their derivative works on different terms, provided the original work is properly cited and the use is non-commercial. See: http://creativecommons.org/ licenses/by-nc/4.0/

(c) Article author(s) (or their employer(s) unless otherwise stated in the text of the article) 2018. All rights reserved. No commercial use is permitted unless otherwise expressly granted.

\section{REFERENCES}

1. Ferlay J, Soerjomataram I, Dikshit R, et al. Cancer incidence and mortality worldwide: sources, methods and major patterns in GLOBOCAN 2012. Int J Cancer 2015;136:E359-E386.

2. AlHW. Australian Cancer Incidence and Mortality (ACIM) books. Canberra: Australian Institute of Health and Welfare, 2014. (accessed 23 Jan 2015).

3. Youlden DR, Cramb SM, Dunn NA, et al. The descriptive epidemiology of female breast cancer: an international comparison of screening, incidence, survival and mortality. Cancer Epidemiol 2012;36:237-48.

4. McKenzie F, Ives A, Jeffreys M. Socio-economic inequalities in survival from screen-detected breast cancer in South West England: population-based cohort study. Eur J Public Health 2012;22:418-22.

5. Panagopoulou P, Gogas H, Dessypris N, et al. Survival from breast cancer in relation to access to tertiary healthcare, body mass index, tumor characteristics and treatment: a Hellenic Cooperative Oncology Group (HeCOG) study. Eur J Epidemiol 2012;27:857-66.

6. Sprague BL, Trentham-Dietz A, Gangnon RE, et al. Socioeconomic status and survival after an invasive breast cancer diagnosis. Cancer 2011;117:1542-51.

7. Leung J, McKenzie S, Martin J, et al. Effect of rurality on screening for breast cancer: a systematic review and meta-analysis comparing mammography. Rural Remote Health 2014;14:2730.

8. Meilleur A, Subramanian SV, Plascak JJ, et al. Rural residence and cancer outcomes in the United States: issues and challenges. Cancer Epidemiol Biomarkers Prev 2013;22:1657-67.

9. Nguyen-Pham S, Leung J, McLaughlin D. Disparities in breast cancer stage at diagnosis in urban and rural adult women: a systematic review and meta-analysis. Ann Epidemiol 2014;24:228-35.

10. Coleman MP, Forman D, Bryant $\mathrm{H}$, et al. Cancer survival in Australia, Canada, Denmark, Norway, Sweden, and the UK, 1995-2007 (the International Cancer Benchmarking Partnership): an analysis of population-based cancer registry data. Lancet 2011;377:127-38.

11. AlHW. Breast cancer in Australia: an overview Cancer series no. 71 . Cat. no. CAN 67. Canberra: Australian Institute of Health \& Welfare, 2012. (accessed 23 Jan 2015).

12. Australian Institute of Health and Welfare. Cancer survival and prevalence in Australia: period estimates from 1982 to 2010. Asia Pac J Clin Oncol 2013;9:29-39.

13. Underhill C, Bartel R, Goldstein D, et al. Mapping oncology services in regional and rural Australia. Aust J Rural Health 2009;17:321-9.

14. Smith T. A long way from home: access to cancer care for rural Australians. Radiography 2012;18:38-42.

15. ABS. Regional population growth, Australia, 2012-13. Canberra: Australian Bureau of Statistics, 2013. (accessed 24 Feb 2015).

16. AlHW. Rural, regional and remote health: a study on mortality (2nd edition) Rural Health Series no.8. Cat. no. PHE 95. Canberra: Australian Institute of Health \& Welfare, 2007. (accessed 24 Feb 2015). 
17. Murphy C, Sabesan S, Steer C, et al. Oncology service initiatives and research in regional Australia. Aust J Rural Health 2015;23:40-8

18. Youl PH, Dasgupta $P$, Youlden $D$, et al. A systematic review of inequalities in psychosocial outcomes for women with breast cancer according to residential location and Indigenous status in Australia. Psychooncology 2016;25:1157-67.

19. Dasgupta P, Baade PD, Youlden DR, et al. Variations in outcomes for Indigenous women with breast cancer in Australia: A systematic review. Eur J Cancer Care 2017;26:e12662.

20. Liberati A, Altman DG, Tetzlaff J, et al. The PRISMA statement for reporting systematic reviews and meta-analyses of studies that evaluate healthcare interventions: explanation and elaboration. $B M$ 2009;339:b2700.

21. University of the Sunshine Coast. Answering a Good Question (PICO). 2015 http://www.usc.edu/hsc/ebnet/ebframe/PICO.htm\#C (accessed 8 May 2015).

22. Wells G, Shea B, O'Connell D, et al. The Newcastle-Ottawa Scale (NOS) for assesing the quality of non-randomised studies in metaanalyses. 2013 http://www.ohri.ca/programs/clinical_epidemiology/ oxford.asp (accessed 20 Feb 2015).

23. Higgins JPT, Green S, eds. Cochrane handbook for systematic reviews of interventions version 5.1.0. The Cochrane Collaboration, 2011. updated March 2011.

24. NHMRC. NHMRC levels of evidence and grades for recommendations for guideline developers: National Health and Medical Research Council, Canberra. 2009 https://www.nhmrc.gov. au/ files nhmrc/file/guidelines/developers/nhmrc levels grades evidence_120423.pdf (accessed 24 Feb 2015).

25. University of York. Systematic Reviews, CRD's guidance for undertaking reviews in health care. York: University of York: Centre for Reviews and Dissemination, 2008. (accessed 24 Mar 2015).

26. Roder D, Zorbas H, Kollias J, et al. Factors predictive of treatment by Australian breast surgeons of invasive female breast cancer by mastectomy rather than breast conserving surgery. Asian Pac J Cancer Prev 2013;14:539-45.

27. AlHW. Rural, regional and remote health: a guide to remoteness classifications. Cat. No. PHE 53. Canberra: Australian Institute of Health and Welfare, 2004.

28. Roder D, Webster F, Zorbas H, et al. Breast screening and breast cancer survival in Aboriginal and Torres Strait Islander women of Australia. Asian Pac J Cancer Prev 2012;13:147-55.

29. Bonett A, Dorsch M, Roder D, et al. Infiltrating ductal carcinoma of the breast in South Australia. Implications of trends in tumour diameter, nodal status and case-survival rates for cancer control. Med J Aust 1990;152:19-23.

30. Chen TY, Morrell S, Thomson W, et al. Survival from breast, colon, lung, ovarian and rectal cancer by geographical remoteness in New South Wales, Australia, 2000-2008. Aust J Rural Health 2015;23:49-56.

31. Clayforth C, Fritschi L, McEvoy SP, et al. Five-year survival from breast cancer in Western Australia over a decade. Breast 2007;16:375-81.

32. Cramb SM, Mengersen KL, Turrell G, et al. Spatial inequalities in colorectal and breast cancer survival: premature deaths and associated factors. Health Place 2012;18:1412-21.

33. Cramb SM, Mengersen KL, Baade PD. Spatio-temporal relative survival of breast and colorectal cancer in Queensland, Australia 2001-2011. Spat Spatiotemporal Epidemiol 2016;19:103-14.

34. Cramb SM, Mengersen KL, Lambert PC, et al. A flexible parametric approach to examining spatial variation in relative survival. Stat Med 2016;35:5448-63.

35. Cramb SM, Moraga P, Mengersen KL, et al. Spatial variation in cancer incidence and survival over time across Queensland, Australia. Spat Spatiotemporal Epidemiol 2017;23:59-67.

36. Dasgupta P, Baade PD, Aitken JF, et al. Multilevel determinants of breast cancer survival: association with geographic remoteness and area-level socioeconomic disadvantage. Breast Cancer Res Treat 2012;132:701-10.

37. Hall S, Holman CD, Sheiner $\mathrm{H}$, et al. The influence of socioeconomic and locational disadvantage on survival after a diagnosis of lung or breast cancer in Western Australia. J Health Serv Res Policy 2004:9(Suppl 2):10-16.

38. Hsieh JC, Cramb SM, McGree JM, et al. Spatially varying coefficient inequalities: evaluating how the impact of patient characteristics on breast cancer survival varies by location. PLoS One 2016;11:e0155086.

39. Mitchell KJ, Fritschi L, Reid A, et al. Rural-urban differences in the presentation, management and survival of breast cancer in Western Australia. Breast 2006;15:769-76.
40. Spilsbury K, Semmens JB, Saunders CM, et al. Long-term survival outcomes following breast cancer surgery in Western Australia. ANZ J Surg 2005;75:625-30.

41. Supramaniam R, Gibberd A, Dillon A, et al. Increasing rates of surgical treatment and preventing comorbidities may increase breast cancer survival for Aboriginal women. BMC Cancer 2014;14:163.

42. Tervonen HE, Aranda S, Roder D, et al. Cancer survival disparities worsening by socio-economic disadvantage over the last 3 decades in new South Wales, Australia. BMC Public Health 2017;17:691.

43. Tracey E, Roder D, Zorbas H, et al. Survival and degree of spread for female breast cancers in New South Wales from 1980 to 2003: implications for cancer control. Cancer Causes Control 2008;19:1121-30.

44. Yu XQ, Luo Q, Kahn C, et al. Temporal trends show improved breast cancer survival in Australia but widening urban-rural differences. Breast 2015;24:524-7.

45. Wilkinson D, Cameron K. Cancer and cancer risk in South Australia: what evidence for a rural-urban health differential? Aust J Rural Health 2004;12:61-6.

46. Taylor R. Breast cancer five-year survival, by New South Wales regions, 1980 to 1991. Aust N Z J Public Health 1997;21:206-10.

47. Hsieh JC-F, Cramb SM, McGree JM, et al. Does geographic location impact the survival differential between screen- and interval-detected breast cancers? Stochastic Environmental Research and Risk Assessment 2016;30:155-65.

48. Hsieh JC-F, Cramb SM, McGree JM, et al. Bayesian spatial analysis for the evaluation of breast cancer detection methods. Aust $N Z$ J Stat 2013;55:351-67

49. Roder D, Zorbas H, Kollias J, et al. Risk factors for poorer breast cancer outcomes in residents of remote areas of Australia. Asian Pac J Cancer Prev 2013;14:547-52.

50. Roder D, Zorbas HM, Kollias J, et al. Analysing risk factors for poorer breast cancer outcomes in residents of lower socioeconomic areas of Australia. Aust Health Rev 2014;38:134-41.

51. Baade PD, Turrell G, Aitken JF. Geographic remoteness, area-level socio-economic disadvantage and advanced breast cancer: a cross-sectional, multilevel study. J Epidemiol Community Health 2011;65:1037-43.

52. Dasgupta P, Youl PH, Aitken JF, et al. Geographical differences in risk of advanced breast cancer: Limited evidence for reductions over time, Queensland, Australia 1997-2014. Breast 2017;36:60-6.

53. Kok DL, Chang JH, Erbas B, et al. Urban-rural differences in the management of screen-detected invasive breast cancer and ductal carcinoma in situ in victoria. ANZ J Surg 2006;76:996-1001.

54. Depczynski J, Dobbins T, Armstrong B, et al. Stage of diagnosis of prostate, breast and colorectal cancer in farm residents compared with other rural and urban residents in New South Wales. Aust $J$ Rural Health 2018;26.

55. Fox PN, Chatfield MD, Beith JM, et al. Factors delaying chemotherapy for breast cancer in four urban and rural oncology units. ANZ J Surg 2013;83:533-8.

56. Lord SJ, Marinovich ML, Patterson JA, et al. Incidence of metastatic breast cancer in an Australian population-based cohort of women with non-metastatic breast cancer at diagnosis. Med J Aust 2012;196:688-92

57. Leung J, Martin J, McLaughlin D. Rural-urban disparities in stage of breast cancer at diagnosis in Australian women. Aust J Rural Health 2016;24:326-32.

58. Luke C, Nguyen AM, Priest K, et al. Female breast cancers are getting smaller, but socio-demographic differences remain. Aust $N$ Z J Public Health 2004;28:312-6.

59. AlHW. BreastScreen Australia: monitoring report 2011-2012. Cat. no. CAN 83. Canberra: Australian Institute of Health and Welfare, 2014. (accessed 24 Feb 2015).

60. Leung J, McKenzie S, Martin J, et al. Longitudinal patterns of breast cancer screening: mammography, clinical, and breast selfexaminations in a rural and urban setting. Womens Health Issues 2014;24:e139-e146.

61. Leung J, Macleod C, McLaughlin D, et al. Screening mammography uptake within Australia and Scotland in rural and urban populations. Prev Med Rep 2015;2:559-62.

62. Barratt AL, Cockburn J, Redman S, et al. Mammographic screening: results from the 1996 National Breast Health Survey. Med J Aust 1997;167:521-4.

63. Sullivan SG, Glasson EJ, Hussain R, et al. Breast cancer and the uptake of mammography screening services by women with intellectual disabilities. Prev Med 2003;37:507-12.

64. Weber MF, Chiew M, Feletto E, et al. Cancer Screening among immigrants living in urban and regional Australia: results from the 45 and up study. Int J Environ Res Public Health 2014;11:8251-66. 
65. Schofield PE, Cockburn J, Hill DJ, et al. Encouraging attendance at a screening mammography programme: determinants of response to different recruitment strategies. J Med Screen 1994;1:144-9.

66. Siahpush M, Singh GK. Sociodemographic variations in breast cancer screening behavior among Australian women: results from the 1995 National Health Survey. Prev Med 2002;35:174-80.

67. Cockburn J, Sutherland M, Cappiello M, et al. Predictors of attendance at a relocatable mammography service for rural women. Aust N Z J Public Health 1997:21:739-42.

68. Hughes JP, Jose DC, Tuch GH, et al. Is Step Down Assessment of screen-detected lesions as safe as workup at a metropolitan assessment centre? Aust N Z J Public Health 2014;38:44-8.

69. O'Byrne AM, Kavanagh AM, Ugoni A, et al. Predictors of nonattendance for second round mammography in an Australian mammographic screening programme. J Med Screen 2000;7:190-4.

70. National Breast Cancer Centre (NBCC). Clinical practice guidelines for management of early breast cancer. 2nd edn. Canberra: Commonwealth of Australia, 2001. (accessed 24 Mar 2015).

71. NBOCC. Guide for women with secondary breast cancer. Surry Hills, NSW: National Breast and Ovarian Cancer Centre, 2010. (accessed 24 Mar 2015).

72. Azzopardi J, Walsh D, Chong C, et al. Impact of geographic location on surgical outcomes of women with breast cancer. ANZ J Surg 2014;84:735-9.

73. Adelson P, Lim K, Churches T, et al. Surgical treatment of breast cancer in New South Wales 1991, 1992. Aust N Z J Surg 1997;67:9-14

74. Martin MA, Meyricke R, O'Neill T, et al. Mastectomy or breast conserving surgery? Factors affecting type of surgical treatment for breast cancer - a classification tree approach. BMC Cancer 2006;6.

75. Mastaglia B, Kristjanson LJ. Factors influencing women's decisions for choice of surgery for Stage I and Stage II breast cancer in Western Australia. J Adv Nurs 2001;35:836-47.

76. Thompson B, Baade P, Coory M, et al. Patterns of surgical treatment for women diagnosed with early breast cancer in Queensland. Ann Surg Oncol 2008;15:443-51.

77. Baade PD, Dasgupta P, Youl PH, et al. Geographical inequalities in surgical treatment for localized female breast cancer, Queensland, Australia 1997-2011: improvements over time but inequalities remain. Int J Environ Res Public Health 2016;13:729.

78. Craft PS, Primrose JG, Lindner JA, et al. Surgical management of breast cancer in Australian women in 1993: analysis of Medicare statistics. Med J Aust 1997;166:626-9.

79. Hill DJ, White VM, Giles GG, et al. Changes in the investigation and management of primary operable breast cancer in Victoria. Med $\mathrm{J}$ Aust 1994;161:110-8.

80. Hall SE, Holman CD, Hendrie DV, et al. Unequal access to breastconserving surgery in Western Australia 1982-2000. ANZ J Surg 2004;74:413-9.

81. Koshy A, Buckingham JM, Zhang Y, et al. Surgical management of invasive breast cancer: a 5-year prospective study of treatment in the Australian Capital Territory and South-Eastern New South Wales. ANZ J Surg 2005;75:757-61.

82. Kricker A, Haskill J, Armstrong BK. Breast conservation, mastectomy and axillary surgery in New South Wales women in 1992 and 1995. Br J Cancer 2001;85:668-73.

83. Hsieh JC, Cramb SM, McGree JM, et al. Geographic variation in the intended choice of adjuvant treatments for women diagnosed with screen-detected breast cancer in Queensland. BMC Public Health 2015;15:1204.

84. Ahern T, Gardner A, Courtney M. Geographical comparisons of information and support needs of Australian women following the primary treatment of breast cancer: a 10-year replication study. Health Expect 2015;18:2678-92.

85. Chong $C$, Walters $D$, de Silva $P$, et al. Initial axillary surgery: results from the BreastSurgANZ Quality Audit. ANZ J Surg 2015;85:777-82.

86. Dasgupta P, Youl PH, Pyke C, et al. Sentinel node biopsy for early breast cancer in Queensland, Australia, during 2008-2012. ANZ J Surg 2017.

87. Morris T, Wetzig N, Sinclair S, et al. Evaluation of implementation of sentinel node biopsy in Australia. ANZ J Surg 2012;82(7-8):541-7.

88. Bell RJ, Robinson PJ, Fradkin P, et al. Breast reconstruction following mastectomy for invasive breast cancer is strongly influenced by demographic factors in women in Victoria, Australia. Breast 2012;21:394-400.

89. Dasgupta P, Youl PH, Pyke C, et al. Geographical disparity in breast reconstruction following mastectomy has reduced over time. ANZ J Surg 2017;87:E183-E187.
90. Flitcroft K, Brennan M, Costa D, et al. Documenting patterns of breast reconstruction in Australia: The national picture. Breast 2016;30:47-53.

91. Roder $\mathrm{D}$, Zorbas $\mathrm{H}$, Kollias $\mathrm{J}$, et al. Factors predictive of immediate breast reconstruction following mastectomy for invasive breast cancer in Australia. Breast 2013;22:1220-5.

92. Hall SE, Holman CD. Inequalities in breast cancer reconstructive surgery according to social and locational status in Western Australia. Eur J Surg Oncol 2003;29:519-25.

93. Lai JK, Martin MA, Meyricke R, et al. Factors associated with short-term hospital readmission rates for breast cancer patients in Western Australia: an observational study. J Am Coll Surg 2007;204:193-200.

94. Ahern T, Gardner A, Courtney M. Exploring patient support by breast care nurses and geographical residence as moderators of the unmet needs and self-efficacy of Australian women with breast cancer: Results from a cross-sectional, nationwide survey. Eur $J$ Oncol Nurs 2016;23:72-80.

95. Campbell D, Khan A, Rankin N, et al. Are specialist breast nurses available to Australian women with breast cancer? Cancer Nurs 2006;29:43-8

96. Eley RM, Rogers-Clark C, Murray K. The value of a breast care nurse in supporting rural and remote cancer patients in Queensland. Cancer Nurs 2008;31:E10-E18.

97. Budden LM, Hayes BA, Buettner PG. Women's decision satisfaction and psychological distress following early breast cancer treatment: a treatment decision support role for nurses. Int $J$ Nurs Pract 2014;20:8-16.

98. Ristevski E, Regan M, Birks D, et al. Communicating about breast cancer: rural women's experience of interacting with their surgeon. Aust J Rural Health 2012;20:22-8.

99. Tulloh BR, Goldsworthy ME. Breast cancer management: a rural perspective. Med J Aust 1997:166:26-9.

100. Lam J, Cook T, Foster S, et al. Examining determinants of radiotherapy access: do cost and radiotherapy inconvenience affect uptake of breast-conserving treatment for early breast cancer? Clin Oncol 2015;27:465-71

101. Collins IM, Lum C, Versace VL. Influence of socioeconomic factors and distance to radiotherapy on breast-conserving surgery rates for early breast cancer in regional Australia; implications of change. Asia Pac J Clin Oncol 2017.

102. Albornoz CR, Cordeiro PG, Hishon L, et al. A nationwide analysis of the relationship between hospital volume and outcome for autologous breast reconstruction. Plast Reconstr Surg 2013;132:192e-200.

103. Gentil J, Dabakuyo TS, Ouedraogo S, et al. For patients with breast cancer, geographic and social disparities are independent determinants of access to specialized surgeons. A eleven-year population-based multilevel analysis. BMC Cancer 2012;12:351.

104. Kong AL, Yen TW, Pezzin LE, et al. Socioeconomic and racial differences in treatment for breast cancer at a low-volume hospital. Ann Surg Oncol 2011;18:3220-7.

105. Gooiker GA, van Gijn W, Post PN, et al. A systematic review and meta-analysis of the volume-outcome relationship in the surgical treatment of breast cancer. Are breast cancer patients better of with a high volume provider? Eur J Surg Oncol 2010;36(Suppl 1):S27-S35.

106. Ess S, Joerger M, Frick H, et al. Predictors of state-of-the-art management of early breast cancer in Switzerland. Ann Oncol 2011:22:618-24.

107. Hershman DL, Richards CA, Kalinsky K, et al. Influence of health insurance, hospital factors and physician volume on receipt of immediate post-mastectomy reconstruction in women with invasive and non-invasive breast cancer. Breast Cancer Res Treat 2012:136:535-45.

108. Roder DM, de Silva P, Zorbas HN, et al. Adherence to recommended treatments for early invasive breast cancer: decisions of women attending surgeons in the breast cancer audit of Australia and New Zealand. Asian Pac J Cancer Prev 2012:13:1675-82

109. National Breast and Ovarian Cancer Centre. Recommendations for follow-up of women with early breast cancer. Surry Hills: National Breast and Ovarian Cancer Centre, 2010. (accessed 24 Mar 2015).

110. Lauby-Secretan B, Scoccianti C, Loomis D, et al. Breast-cancer screening--viewpoint of the IARC Working Group. N Engl J Med 2015;372:2353-8.

111. Welch HG, Prorok PC, O'Malley AJ, et al. Breast-cancer tumor size, overdiagnosis, and mammography screening effectiveness. N Engl J Med 2016;375:1438-47.

112. Breast Cancer Network Australia. Women living in rural and remote areas. 2017 https://www.bcna.org.au/about-us/advocacy/position- 
statements/women-living-in-rural-and-remote-regions/ (accessed 28 Nov 2017)

113. Wilcoxon H, Luxford K, Saunders C, et al. Multidisciplinary cancer care in Australia: a national audit highlights gaps in care and medico-legal risk for clinicians. Asia Pac J Clin Oncol 2011;7:34-40.

114. Cancer Council Australia. Optimal care pathway for women with breast cancer [web page]. 2017 http://www.cancer.org.au/healthprofessionals/optimal-cancer-care-pathways.html (accessed 29 Nov 2017).

115. National Institute for Clinical Excellence. NICE Clinical Guidelines [CG80]- Early and locally advanced breast cancer: diagnosis and treatment: United Kingdom National Collaborating Centre for Cancer, 2009. updated Feb 2009. (accessed 15 Jan 2016).

116. Senkus E, Kyriakides S, Ohno S, et al. Primary breast cancer: ESMO Clinical Practice Guidelines for diagnosis, treatment and follow-up. Ann Oncol 2015;26(suppl 5):v8-v30.

117. McKevitt EC, Dingee CK, Warburton R, et al. Coordination of radiologic and clinical care reduces the wait time to breast cancer diagnosis. Curr Oncol 2017;24:388-e93

118. Prades J, Remue E, van Hoof $\mathrm{E}$, et al. Is it worth reorganising cancer services on the basis of multidisciplinary teams (MDTs)? A systematic review of the objectives and organisation of MDTs and their impact on patient outcomes. Health Policy 2015;119:464-74.

119. Taylor C, Shewbridge A, Harris J, et al. Benefits of multidisciplinary teamwork in the management of breast cancer. Breast Cancer 2013;5:79-85.

120. Wong A, Snook K, Brennan M, et al. Increasing breast reconstruction rates by offering more women a choice. ANZ J Surg 2014;84:31-6.

121. Rogers MJ, Matheson L, Garrard B, et al. Comparison of outcomes for cancer patients discussed and not discussed at a multidisciplinary meeting. Public Health 2017;149:74-80.

122. AlHW. Hospital resources 2015-16: Australian hospital statistics. Health services series no. 78. Cat. no. HSE 190. 2017 https://www. aihw.gov.au/getmedia/d37a56cb-dc6b-4b28-a52f-8e00f606ce67/ 21035.pdf.aspx?inline=true (accessed 4 Dec 2017).

123. National Breast and Ovarian Cancer Centre. Recommendations for the management of early breast cancer in women with an identified BRCA1 or BRCA2 gene mutation or at high risk of a gene mutation. Surry Hills: National Breast and Ovarian Cancer Centre, 2014 (accessed 24 Mar 2015).

124. National Breast and Ovarian Cancer Centre Recommendations for use of Sentinel node biopsy in early (operable) breast cancer National Breast and Ovarian Cancer Centre. Surry Hills: National Breast and Ovarian Cancer Centre, 2008. (accessed 24 Mar 2015).

125. The Royal Australian and New Zealand College of Radiologists. Planning for the Best: tripartite National Strategic Plan for Radiation Oncology 2012-2022, version 1. Sydney, Australia: The Royal
Australian and New Zealand College of Radiologists, 2012 (accessed 25 Apr 2015)

126. Australian Institute of Health and Welfare. Radiotherapy in Australia 2015-16. HSE 191. Canberra: Australian Institute of Health and Welfare, 2017. (accessed 20 Nov 2017).

127. Butler SM. Changes to radiotherapy utilisation in Western NSW after the opening of a local service. J Med Radiat Sci 2017;64:251-8.

128. Sharma DK, Vangaveti VN, Larkins S. Geographical access to radiation therapy in North Queensland: a retrospective analysis of patient travel to radiation therapy before and after the opening of an additional radiotherapy facility. Rural Remote Health 2016;16:3640.

129. Darby S, McGale P, Correa C, et al. Effect of radiotherapy after breast-conserving surgery on 10-year recurrence and 15-year breast cancer death: meta-analysis of individual patient data for 10,801 women in 17 randomised trials. Lancet 2011;378:1707-16.

130. McGale P, Taylor C, Correa C, et al. Effect of radiotherapy after mastectomy and axillary surgery on 10-year recurrence and 20-year breast cancer mortality: meta-analysis of individual patient data for 8135 women in 22 randomised trials. Lancet 2014;383:2127-35.

131. Olson RA, Nichol A, Caron NR, et al. Effect of community population size on breast cancer screening, stage distribution, treatment use and outcomes. Can J Public Health 2012;103:46-52.

132. Dragun AE, Huang B, Tucker TC, et al. Disparities in the application of adjuvant radiotherapy after breast-conserving surgery for early stage breast cancer. Cancer 2011;117:2590-8.

133. Ess S, Savidan A, Frick H, et al. Geographic variation in breast cancer care in Switzerland. Cancer Epidemiol 2010;34:116-21.

134. Bride MBM, Neal L, Dilaveri CA, et al. Factors associated with surgical decision making in women with early-stage breast cancer: a literature review. J Womens Health 2013;22:236-42.

135. Markossian TW, Hines RB. Disparities in late stage diagnosis, treatment, and breast cancer-related death by race, age, and rural residence among women in Georgia. Women Health 2012;52:317-35.

136. Zhong T, Fernandes KA, Saskin R, et al. Barriers to immediate breast reconstruction in the canadian universal health care system. Journal of Clinical Oncology 2014;32:2133-41.

137. Baade PD, Yu XQ, Smith DP, et al. Geographic disparities in prostate cancer outcomes - review of international patterns. Asian Pacific Journal of Cancer Prevention 2015;16:1259-75.

138. Chawla N, Butler EN, Lund J, et al. Patterns of colorectal cancer care in Europe, Australia, and New Zealand. JNCI Monographs 2013;2013:36-61.

139. AlHW. Australian cancer database. Canberra: Australian Institute of Health \& Welfare, 2017. (accessed 12 Nov 2017).

140. Sabesan S, Kelly J. Are teleoncology models merely about avoiding long distance travel for patients? Eur J Cancer Care 2014;23:745-9. 